\title{
Laser Technology Improving Wireless Communication: A Comprehensive Study on Laser Communicator
}

\author{
"Sakshi Taaresh Khanna, Kamal Sharma \\ Department of Computer Science, Ram Lal Anand College/ University of Delhi, India \\ Department of Computer Science, Ram Lal Anand College/ University of Delhi, India \\ Corresponding Author: Sakshi Taaresh Khanna
}

\begin{abstract}
Since the origin of wireless communication it has gained huge popularity and acceptance in the whole world, providing a setup which uses minimum amount of cables for transmission of data. There has been an explosive growth in the wireless communications over the last few decades in its applications cellular telephony, wireless internet and wireless home networking arenas. As we have advanced so has the data processed and transmitted has increased exponentially leading to Big Data problems. The wireless media is now becoming less efficient in handling large amounts of data signaling to a new innovation in communications. This paper is intended to provide the reader with an overview of innovation that has been attained with the help of lasers to transmit sound and also elaborates on how it is being used along with the future developments.
\end{abstract}

Keywords: Laser power transmission, Receiver, Transmitter, Wireless Network

Date of Submission: 03-07-2017

Date of acceptance: 15-07-2017

\section{Introduction}

In 1864 James Clerk Maxwell proved the existence of electromagnetic waves [14] and after deep research and development, the ability to communicate by using electromagnetic waves was developed and now has been deeply integrated into many aspects. But as we have advanced we are beginning to see the problems of wireless networks which are as follows:

\subsection{Signal fading}

Unlike wired media, signals transmitted over a wireless medium may be distorted or weakened because they are propagated over an open, unprotected, and ever changing medium with irregular boundary. Besides, the same signal may disperse and travel on different paths due to reflection, diffraction, and scattering caused by obstacles before it arrives at the receiver. The dispersed signals on different paths may take different times to reach the destination. Thus, the resultant signal after summing up all dispersed signals may have been significantly distorted and attenuated when compared with the transmitted signal. The receiver may not recognize the signal and hence the transmitted data cannot be received. This unreliable nature of the wireless medium causes a substantial number of packet losses.[1]

\subsection{Mobility}

Without the constraints imposed by the wired connections among devices, all devices in a wireless network are free to move. To support mobility, an ongoing connection should be kept alive as a user roams around. In an infrastructure network, a handoff occurs when a mobile host moves from the coverage of a base station or access point to that of another one. A protocol is therefore required to ensure seamless transition during a handoff. This includes deciding when a handoff should occur and how data is routed during the handoff process. In some occasions, packets are lost during a handoff. In an ad hoc network, the topology changes when a mobile host moves. This means that, for an ongoing data communication, the transmission route may need to be recomputed to, cater for the topological changes. Since an ad hoc network may consist of a large number of mobile hosts, this imposes a significant challenge on the design of an effective and efficient routing protocol that can work well in an environment with frequent topological changes.[1]

\subsection{Power and energy}

A mobile device is generally handy, small in size, and dedicated to perform a certain set of functions; its power source may not be able to deliver power as much as the one installed in a fixed device. When a device is allowed to move freely, it would generally be hard to receive a continuous supply of power. To conserve energy, a mobile device should be able to operate in an effective and efficient manner. To be specific, it should 
be able to transmit and receive in an intelligent manner so as to minimize the number of transmissions and receptions for certain communication operations. [1][3]

\subsection{Data Rate}

Improving the current data rates to support future high speed applications is essential, especially, if multimedia service are to be provided. Data rate is a function of various factors such as the data compression algorithm, interference mitigation through error-resilient coding, power control, and the data transfer protocol. Therefore, it is imperative that manufacturers implement a well thought out design that considers these factors in order to achieve higher data rates. Data compression plays a major role when multimedia applications are to be supported.[1]

\subsection{Security}

Mobility of users increases the security concerns in a wireless network [4]. Current wireless networks employ authentication and data encryption techniques on the air interface to provide security to its users. The IEEE 801.11 standard [5] describes wired equivalent privacy (WEP) that defines a method to authenticate users and encrypt data between the PC card and the wireless LAN access point.[1]

\subsection{Quality of Service (QoS)}

Quality of Service is a measure of network performance that reflects the network's transmission quality and service availability. For each flow of network traffic, QoS can be characterized by four parameters: Reliability, Delay, Jitter, and Bandwidth.[1]

\subsection{Objective}

\section{Sound transfer using LASER (Laser Communicator)}

- Transmission of sound using laser light as the carrier which is accomplished by a transmitter.

- Reception of the signal at the receiver and then conversion to the original signal.

2.2 HARDWARE COMPONENTS REQUIRED

\section{- Transmitter}

- Receiver

\subsection{1 for Transmitter.}

- A laser(in this assignment, red is chosen, $5 \mathrm{~mW}, 650 \mathrm{~nm}$, class III A pen laser)

- 3 AA batteries 1.5 volts each along with holder (4.5 volts total)

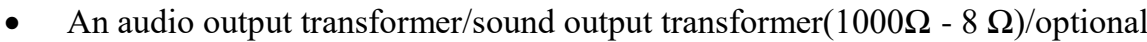

- Clip leads

- Aux cable with striped ends

- Sound source(mike/ radio/ phone)

\subsection{2 for Receiver.}

- Solar panel (1 watt)

- Clip leads

- Amplifier(radio with aux in)/ a speaker along with aux jointer

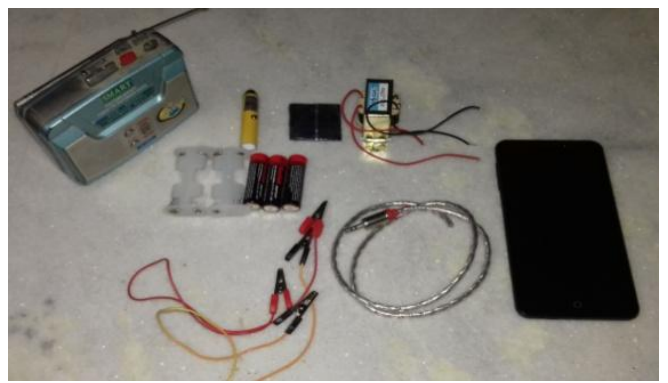

Figure 1: Components required for sound transfer using laser

\subsection{Arrangement of hardware at transmitter side}

Remove the cells of the batter and run the clip leads between the battery, transformer, aux cable and the laser as shown in the circuit diagram. 


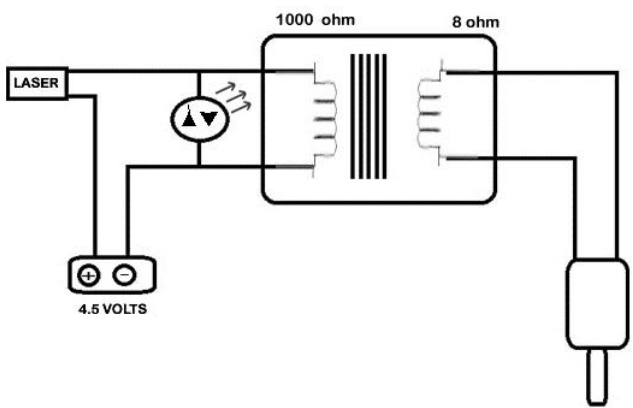

Figure 2:Transmitter

If the transformer is not available, then connect the laser, batteries and the aux cable without the transformer. When the batteries are removed a spring can be seen, this is the negative end, connect this end to the negative terminal of the battery and connect the positive end of the battery to the body of the laser through jumpers. If a 3 cell holder is not available then you can modify either 2 two cell holders or a 4 cell holder.

\subsection{Arrangement of hardware at receiver side}

The solar panel is connected to an aux cable which will be connected to an amplifier (radio) as shown in the circuit diagram. The receiver can also be connected to a speaker via aux jointer.

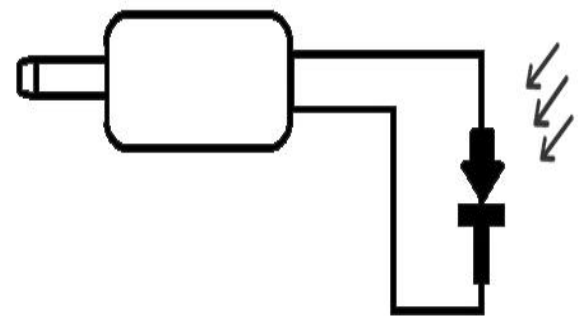

Figure 3. Receiver

The negative end of the solar panel is connected to the negative end of the aux i.e. the wire which is connected to the tip of the aux connector of the cable.

\subsection{Working of Laser Communicator}

After making connections through the jumpers first test the transmitter by checking whether the laser is working a multimeter can be used to check all the connections. Similarly check the receiver with the help of multimeter. It will show the reading of the voltage output of the solar panel. The laser used gives a change of 0.2 volts when directed towards the solar panel.

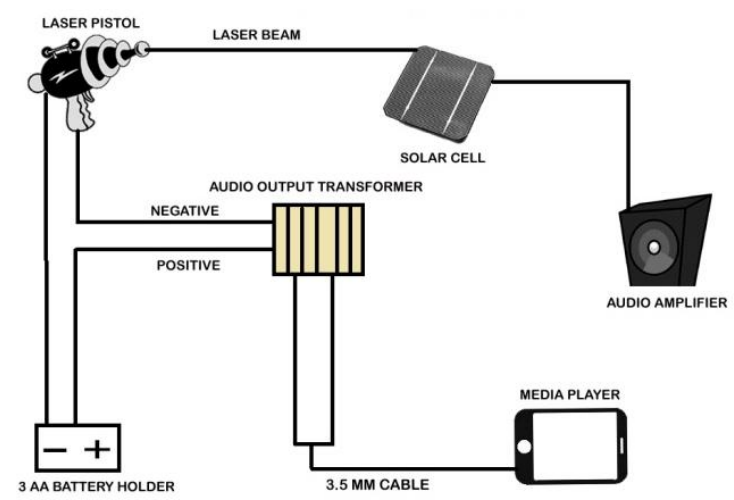

Figure 4. Simple Laser Communication

The sound source is taken to be the music played on a mobile device (any other device such as a radio can also be used). When the song is played it travels as electrical signal to the audio output transformer via the aux cable. The audio output transformer amplifies the electrical signals and then delivers it to laser. The sound is then 
transmitted in the form of light. At the receiving end, the changes in the voltage are sensed by the solar panel and are passed on to the amplifier as changes in voltage. These changes in voltage are converted to sound which is audible via the speaker.

\subsection{Performance}

This technique will give data rates of $622 \mathrm{Mbps}$ (as tested by NASA).[15] The upload speed will be of 20Mbps (as tested by NASA).[15] The performance is directly proportional to the intensity of the laser and inversely proportional to the distance between the sender and receiver. The weather play a very vital role, this technique works best in clear conditions.

\subsection{Advantages}

With this new technique we can transmit any kind of data much faster safer and at very high transfer rates which are out of reach of the R.F. spectrum. As compared to R.F. devices the circuitry used is very less and will take very less space. It is the next generation which will take over fiber optics, R.F. transmission. Its best use will be in space missions and defense systems.

\subsection{Military}

\section{Laser Applications in different fields}

\subsubsection{In Laser Range Finder}

To knock down an enemy tank, it is necessary to range it very accurately. Because of its high intensity and very low divergence even after travelling quite a few kilometers, laser is ideally suited for this purpose. The laser range finders using neodymium and carbon dioxide lasers have become a standard item for artillery and tanks. These laser range finders are light weight and have higher reliability and superior range accuracy as compared to the conventional range finders. The laser range finder works on the principle of radar. It makes use of the characteristic properties of the laser beam, namely, monochromaticity, high intensity, coherency, and directionality. A collimated pulse of the laser beam is directed towards a target and the reflected 1ight from the target is received by an optical system and detected. The time taken by the laser beam for the to and fro travel from the transmitter to the target is measured. When half of the time thus recorded is multiplied by the velocity of light, the product gives the range, i.e., the distance of the target.[17]

The laser range finder is superior to microwave radar as the former provides better collimation or directivity which makes high angular resolution possible. Also, it has the advantage of greater radiant brightness and the fact that this brightness is highly directional even after travelling long distances, the size of the emitting system is greatly reduced. The high monochromaticity permits the use of optical band pass filter in the receiver circuit to differentiate between the signal and the stray light noise. [17]

\subsubsection{Laser Bomb Designators}

The military has enjoyed a considerable amount of success in developing laser devices for use on the battlefield. These devices, which greatly improve the accuracy of normal conventional weapons, include range finders and bomb designators. A range finder calculates the distance, or range, to a desired target by measuring how long a small burst of laser light takes to travel to the target. This practical tool can be either handheld or mounted on a tank. Obviously, if a soldier knows the exact distance to his target, he has a much better chance of hitting it. By the mid-1970s Hughes Aircraft was building more than $\$ 50$ million worth of laser range finders for the U.S. military each year.[16]

A laser bomb designator works by shining a low-powered laser beam at the desired target. After the target has been designated, a bomb is released, either from an airplane or from a ground-based missile; this is known as a "smart" bomb because it carries a sensor that can detect the laser beam and use it to home in on and destroy the target. The military first used such devices on the battlefield in 1972 during the Vietnam War, and much improved versions proved highly successful in the Persian Gulf War (in 1991) and especially in the police action against terrorists in Afghanistan (in 2001).[16]

\subsubsection{Simulating battle conditions}

Lasers have also proved to be successful in simulating mock battles that are staged to give soldiers practice for the real thing. Before lasers, these simulations had not been as realistic as military officials would have liked. Obviously, the soldiers on opposing teams could not really fire at each other, so referees had to decide who had or had not been "hit." Their decisions naturally involved a certain amount of human error.[16]

In laser battle simulations, soldiers fire special guns that shoot bursts of light. Sensors are attached to each soldier who fights in the battle; such sensors are also attached to tanks, trucks, or any other vehicles used in the mock fighting. When a burst of light is fired and hits a sensor on an "enemy" soldier, the sensor registers the light and everyone knows immediately that the soldier is "dead." They know when a tank or truck has been 
destroyed because the sensors mounted on vehicles give off a cloud of smoke when hit. Many companies now build such battle simulators, which are used by armies all over the world.[16]

\subsubsection{Underwater Communication}

Another dramatic use for the military laser is in the area of submarine (underwater) communications. Submarines often patrol in enemy waters, and in the past the only way an admiral could get a message to a sub was by using ordinary radio. But this has two serious disadvantages: First, radio waves do not travel well underwater and require large antennas to broadcast those long distances. Second, there is always the risk that the enemy will pick up the signal, which immediately reveals the sub's location and exposes it to danger.[16]

To send a message to a submarine the navy uses a laser that gives off a monochromatic beam of bluegreen light. This particular shade of blue-green light travels easily through ocean water. The beam carrying the message is transmitted to a satellite orbiting high above the ocean. The satellite then relays the beam down to the sub, which is equipped with a special receiver that registers only blue-green light. In less than a second the sub's computer decodes the signal so the crew can read the message.[16]

If there are any enemy on lookouts nearby, it is unlikely they will know about the signal beam, which the satellite flashes for only a few millionths of a second. This is not enough time for the lookouts to see the beam with their naked eyes. And even if they have a receiver that detects laser light, it has to be tuned to receive the exact shade of blue-green in the beam. Their receiver also has to be underwater near the sub because that is where the satellite aims the beam. Meeting these conditions would be very difficult for the lookouts, so chances are good that the sub will get the message and still remain undetected.[16]

\subsection{Communications}

A very useful and interesting application of laser is in the field of communications, which takes advantage of its wide bandwidth and narrow beam width over long distances. The laser beams can be created in a range of wavelengths from the ultraviolet to the infrared regions of the electromagnetic spectrum. The color of the emitted light is relatively not important. The infrared region is preferred by the military, as it is more difficult to detect.[17]

The advent of semiconductor lasers has made possible the use of lasers for signal transmission. They are excited directly by electric current to yield a laser beam in the invisible infrared region. A particular aspect of laser transmission, which makes it preferable to the ordinary radio waves for military purposes is the strict secrecy provided by the narrow beam width.[17]

Since no unwanted reception outside the narrow bundles of rays is possible, a high degree of secrecy can be maintained between two points, and thus, an interception-proof communication network can be realized. Besides, laser communication system is immune from jamming and from interference by spurious radio noise.[17]

The optical laser has a great potential for use in long distance communication. Since the capacity of a communication channel is proportional to the frequency band width, at optical frequencies, the information carrying capacity is many times more than that is possible at lower frequencies. This and the fact that the laser is a generator of highly coherent beams which are powerful and sharply directed, make it ideally suited for communications. [17] The laser communication through the atmospheric medium is effective only in clear weather conditions, with no obstacles interrupting the beam between the transmitting and the receiving stations [17].

\subsection{Wireless power driven propulsion}

Laser or microwave-driven acceleration by photon reflection has been proposed for propelling spacecraft for science missions to the outer solar system and even to nearby stars. In principle, such wireless beam driven probes have the advantage that energy is used for the acceleration of only the payload (and the receiving/ reflecting structure usually called a "sail") but not the propelling beam generator [2].

\subsection{LCRD}

NASA successfully tested the LCRD (Laser Communication Relay Demonstration) for transmission of data 10 times faster than the conventional R.F. method and will launch a fully operational module in 2017 making a giant leap towards high transmission rates. [18]

\subsection{TALON}

Harris, an American communications, defense contractor and IT services has developed TALON (Tactical Line-of- sight Operational Network) with the help of which navy ships can communicate at high speeds with each other, it also provides high speed video conferencing and has been tested up to 53 miles in the sea. [19] 


\subsection{Fully independent rover vehicle}

In 2002 and 2003, a demonstration of ground to ground wireless power transmission via laser to a small, otherwise fully independent rover vehicle equipped with photovoltaic cells was performed by Steinsiek and Schậfer ,as a first step towards the use of this technology for powering airships and further in the future lunar surface rovers. [6] The experiment was based on a green, frequency-doubled Nd:YAG laser at only a few Watts. It included the initiation and supply of the rover including a micro-camera as payload as well as the pointing and tracking of the moving rover over a distance up to $280 \mathrm{~m}$ by applying active control loops.[2](Fig 5)

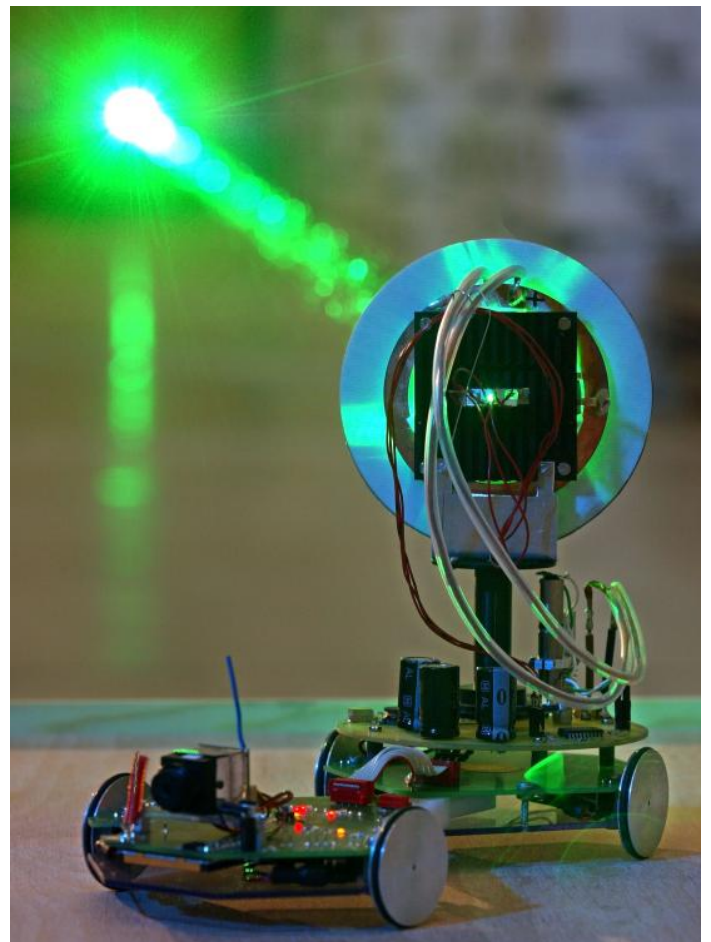

Figure 5: EADS developed, fully laser powered autonomous rover. (Source: EADS)) [10][11]

Recently, similar experiments, however focusing less on the beam control and beam steering aspects but rather on the total transmitted power levels have been carried out in the frame of a context related to space elevators, organized and co-funded by NASA. Ground-based lasers have been used to power small PV-covered "climbers" attached to a tether with the objective to achieve maximum climbing speeds [2][7][8].

\subsection{Laser power transmission}

The use of laser based wireless power transmission was revisited in the early 1990s by Landis. [9][10] Since several years, the Japanese space agency JAXA (Japan Aerospace eXploration Agency) is pursuing a solid and targeted R\&D programme towards the development of space based solar power stations, including as the two main technical options the microwave and laser based concepts. New designs and laser system options have been proposed.[2]

The JAXA proposed laser based system is based on direct solar pumped lasers using a Nd:YAG crystal. A reference system has been designed, delivering in its full configuration $1 \mathrm{GW}$. The entire system would be built in a highly modular way, with individual modules of $100 \mathrm{~m}$ x $200 \mathrm{~m}$ primary mirrors and an equally large radiator system as base unit delivering $10 \mathrm{MW}$ each and stacked to a total length of $10 \mathrm{~km}$ in orbit.(Fig 6, Fig 7, Fig 8 ) [2][11][12]

In 2004, JAXA and the Osaka based Institute for Laser Technology have successfully performed an experiment with direct solar pumped laser beam (using simulated solar light and a fiber laser medium made from a neodymium-chrome doped YAG (Nd-Cr:YAG) crystal and disc type bulk crystal) with conversion efficiencies from the input power to the output laser power with 37\%.[13][2] 


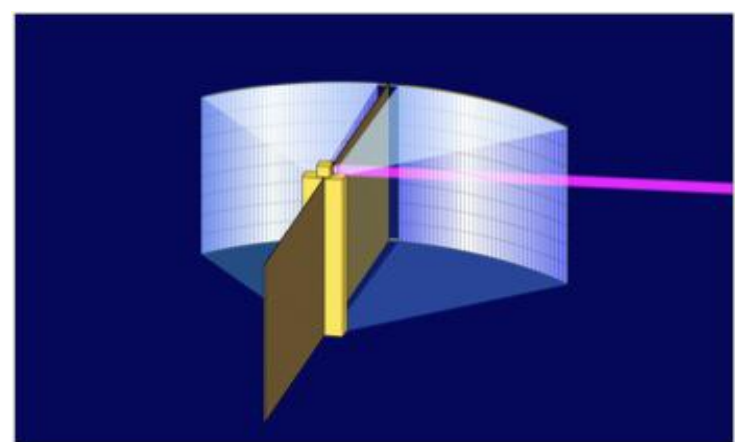

Figure 6. JAXA L-SPS 100x200 m reference unit delivering $10 \mathrm{MW}$ via direct solar pumped lasers. (Source: JAXA) [11][12]

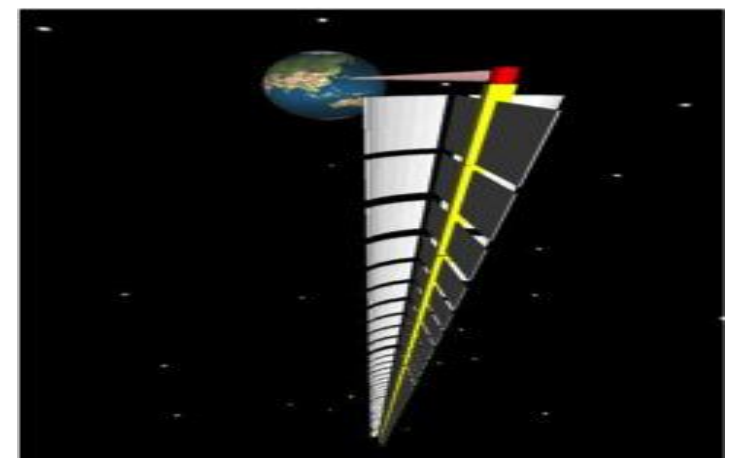

Figure 7. JAXA L-SPS fully deployed reference system delivering $1 \mathrm{GW}$ via direct solar pumped lasers. (source: JAXA), deployed in the orbit of Earth, made of $10 \mathrm{MW}$ unit each, Spread in $10 \mathrm{Kms}$ )[11][12]

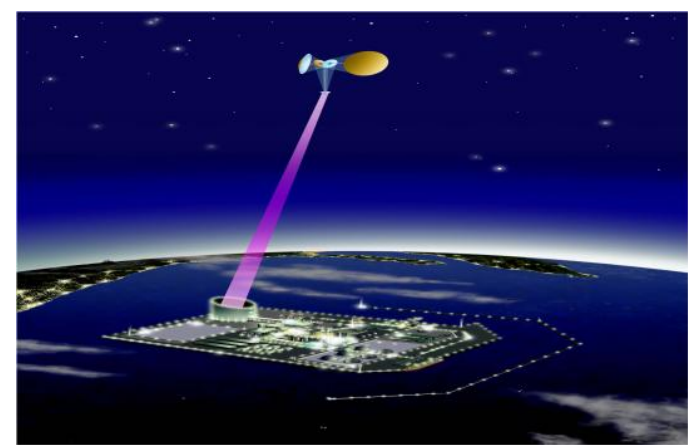

Figure 8. JAXA L-SPS system transmitting power with the help of laser to the station present on the surface of the Earth. (Source: JAXA)[11][12]

\section{Conclusion}

Laser communicator can be applied to send data from one computer to another at speeds which the wireless networks will not be able to provide. Another network which this technique will surpass is the fiber optical network, as this technique will minimize the setup physically and also financially. The implementation of any of these systems in an inter-satellite link will require a substantial development effort. The strengths and weaknesses of the various types of lasers presently available for laser communications should be carefully considered. Based on existing laser's characteristics, the GalAs system, especially the full-bandwidth, direct detection system is the most attractive for inter satellite links because of its inherent simplicity and the expected high level of technological development.

The system and component technology necessary for successful inter satellite link exists today. The growing requirements for the efficient and secure communications has led to an increased interest in the operational deployment of laser cross-links for commercial and military satellite systems in both low earth and geo-synchronous orbits. With the dramatic increase in the data handling requirements for satellite communication services, laser inter satellite links offer an attractive alternative to RF with virtually unlimited potential. Till now data transfer took place either through wireless networks (Wi-Fi) or through wired networks (cables). The Laser technique aims to change the media of communication and at the same time keep in consideration cost constraints and also provide better data rates. Also Cryptanalysis techniques can be applied 
for added security to the data. The basic concept remains the same as that of the Laser communicator. Input is taken from the pc and converted to Morse code sot that it will be easy to convert the 'dot' and 'dash' to voltages and then used to power up the laser. This laser will be directed to the target pc having a receiver of ether photodiode or solar panel to capture the light and sense the voltages and pass on to the application as Morse code and then further decoded to the information. The data rate is much greater than wireless mediums according to initial research. All Computers of a lab can be connected to each other through a direct online link with its help without using any cables and also without compromising on the speeds.

\section{References}

[1] http://www.ijarcsse.com/docs/papers/April2012/Volume_2_issue_4/V2I40056.pdf. International Journal of Advanced Research in Computer Science and Software Engineering Research Paper: Research Issues in Wireless Networks.

[2] [.Concepts for wireless energy transmission via laser by Leopold Summerer, Oisin Purcell. (http://www.spaceward.org/elevator2010-pb)

[3] H. Singh and S. Singh, -Energy Consumption of TCP Reno, Newreno, and SACK in Multi-Hop Wireless Networks II ACM SIGMETRICS Perf. Evaluation Rev., vol. 30, no. 1, June 2002, pp. 206-216.

[4] Chip Craig J. Mathias Principal, Farpoint Group COMNET 2003 -Wireless Security: Critical Issues and Solutions\| 29 January 2003

[5] IEEE 802.11-1999, IEEE Standard for Local and Metropolitan Area Networks Specific Requirements - Part 11: Wireless LAN Medium Access Control (MAC) and Physical Layer (PHY) Specifications, June 12, 1999.

[6] Frank Steinsiek, W. P. Foth, K. H. Weber, Christian Schäfer, and H. J. Foth. Wireless power transmission experiment as an early contribution to planetary exploration missions. In IAC 2003. IAF, Oct. 2003.

[7] The space elevator - from concept to reality, December 2008. http://www.spaceelevator.com (accessed December2008).

[8] Spaceward Foundation. Power beaming (climber)competition http://www.spaceward.org/elevator2010-pb (accessed December 08).

[9] G. A. Landis. Moonbase night power by laser illumination. Journal of Propulsion and Power, 8, 1992.

[10] G. A. Landis. Applications for space power by laser transmission. In Proceedings SPIE, volume 2121, pages 252-255, 1994.

[11] Masahiro Mori, H. Kagawa, and Y. Saito. Current status of a study on hydrogen production with space solar power systems (SSPS). In L. Summerer, editor, SPS'04 Conference - Solar Power from Space, Granada, Spain, June 30 - July 22004 . ESA.

[12] Hiroaki Suzuki, Tatsuhito Fujita, and Masahiro Mori. Technology demonstration and elemental technology development of space solar power systems. In Proceedings of the IAC, number IAC-06-C3.2.4. IAF, 2006.

[13] Masahiro Mori. Medium efficiently converts solar rays into laser beams. The Japan Times, September, 92004.

[14] http://www.wirelesscommunication.nl/reference/chaptr07/history.htm

[15] https://www.nasa.gov/sites/default/files/llcdfactsheet.final_.web_.pdf

[16] http://www.scienceclarified.com/scitech/Lasers/Military-Applications-of-Lasers.html

[17] http://electrofriends.com/projects/basic-electronics/laser-communication-system/2/

[18] https://www.nasa.gov/mission_pages/tdm/lcrd/index.html

[19] https://www.harris.com/press-releases/2014/03/video-exelis-laser-communications-system-connects-deployed-forces-in-any

IOSR Journal of Computer Engineering (IOSR-JCE) is UGC approved Journal with Sl. No. 5019, Journal no. 49102.

Sakshi Taaresh Khanna. "Laser Technology Improving Wireless Communication: A Comprehensive Study on Laser Communicator." IOSR Journal of Computer Engineering (IOSR-JCE) 19.4 (2017): 26-33 